\title{
Características físico-químicas de suelos con relación a su conformación estructural
}

\section{Physical chemical properties of soils in relation to their structural formation}

\author{
Claudia Díaz Mendoza ${ }^{1}$, Cristna Herrera Atencio ${ }^{2}$, Katerin Prada Sánchez ${ }^{3}$.
}

\section{Resumen}

Palabras

Claves:

Suelo, estructural,

físico-química,

humedad, materi orgánica.
Keywords:

Soil, structural, ysicochemical organic matter

humidity, unit weight.

Objetivo: identificar las características físico-químicas del suelo, así como relacionarlas con su comportamiento estructural y las condiciones ambientales, a fin de que sirvan de insumo en diagnósticos ambientales de suelos.

Metodología: el método desarrollado partió de la extracción de muestras superficiales de suelos de manera aleatoria y con profundidades menores a $0.3 \mathrm{~m}$. Las muestras fueron transportadas al laboratorio, donde se les practicaron ensayos de humedad natural, materia orgánica, clasificación de suelos y peso unitario. Luego se realizó un análisis estadístico.

Resultados y conclusiones: de acuerdo con el grupo de suelos identificados, cohesivos y granulares, estos presentan condiciones que pueden incidir en comportamientos ambientales y susceptibilidad en aspectos de fertilidad o eventuales casos de contaminación.

\begin{abstract}
Objective: to identify the physical-chemical characteristics of the soil, as well as to relate them to their structural behavior and environmental conditions, in order to serve as an input in soil environmental diagnostics.

Methodology: the developed method started from the extraction of superficial samples of soils in a random way and with depths less than $0.3 \mathrm{~m}$. The samples were transported to the laboratory, where they were tested for natural moisture, organic matter, soil classification and unit weight. Then a statistical analysis was performed.

Results and conclusions: according to the group of identified, cohesive and granular soils, these present conditions that can affect environmental behaviors and susceptibility in aspects of fertility or eventual cases of contamination.
\end{abstract}

1 Grupo de Investigación Ambiental

2 Autor de correspondencia, Fundación Universitaria Tecnológico Comfenalco Cartagena, Correo electronico:cdiaz@tecnologicocomfenalco.edu.co

3 Fundación Universitaria Tecnológico Comfenalco. Cartagena, Colombia. Correo electronico: Itapia2@areandina.edu.co

Pág 58 Como citar este artículo: C. Díaz, C. Herrera, y K. Prada "Características físico químicas de suelos con relación a su conformación estructural",Investigación e Innovación en Ingenierias, vol. 6, n. 1, pp. 58 - 69., 2018.

DOI: https://10.17081/invinno.6.1.2775 


\section{Introducción}

Para realizar un diagnóstico ambiental, es necesario tener en cuenta los componentes de agua, aire y suelo, entre otros aspectos. En el caso del suelo, gran parte de los diagnósticos que se realizan buscan identificar la susceptibilidad a la contaminación, a procesos de desertización y degradación o, en algunos casos, su estabilidad fértil en procesos de productividad.

En el proyecto realizado, se evalúan condiciones de humedad natural, granulometría, límites de consistencia, material orgánico y peso unitario, para evaluar posibles comportamientos ambientales derivados de sus características físico-químicas.

La materia orgánica presente en el suelo es considerada un indicador de salud del mismo y tiene efectos positivos en la sostenibilidad del sistema productivo. Este nivel de materia orgánica depende del clima, del suelo y del manejo del mismo [1]; en tanto que la heterogeneidad de sus propiedades afecta la distribución del contenido de humedad, que se materializa en variaciones en la textura, materia orgánica y estructura, propiedades todas que controlan el movimiento del flujo de agua en el perfil edáfico. Como indican Hupet y Vanclooster [2], para algunos autores la textura es uno de los factores que mayor control ejerce sobre la humedad del suelo. Esta influencia varía según el contenido de humedad y es más acusada conforme esta aumenta $[3,4,5]$. El peso unitario gravimétrico de un sólido es definido como la masa de un suelo por unidad de volumen. Por su parte, el peso unitario del suelo varía de acuerdo con el contenido de agua que tenga, clasificándose en: húmedo (no saturado), saturado y seco.

En este proyecto se identificaron características propias del suelo a nivel físico-químico, relacionadas con su comportamiento estructural y las condiciones ambientales. 


\section{Metodología}

El proyecto se desarrolló por fases, así:

Fase I: En esta se realizó una revisión bibliográfica en la temática específica del proyecto; así como el análisis y elección de los parámetros a estudiar.

Fase II: Incluyó la recuperación de muestras de suelo a nivel superficial y de manera aleatoria, con profundidades menores a $0.30 \mathrm{~m}$. Las muestras al laboratorio se trasladaron mediante cadena de custodia y se realizaron ensayos de laboratorio a 60 muestras, de diferente conformación estructural. Los parámetros evaluados fueron: humedad natural (INVIAS I.N.V. E-122-07), análisis granulométricos (ASTM D422) y materia orgánica (MO) por calcinación. Este último es en sí un método cuantitativo más conocido como pérdida por ignición, en el cual se cuantifica directamente el contenido de $\mathrm{MO}$ por medio de la pérdida de peso de una muestra de suelo al someterla a elevadas temperaturas. En este caso, para estimar COT a partir de la MO también se debe utilizar el factor Van Bemmelen [5], (I.N.V. E - 121 - 07; AASHTO T 267 - 86 (2004) y conocer el peso unitario o Densidad Suelta (I.N.V. E - 217 - 07).

Fase III: Durante esta se realizó un análisis estadístico descriptivo multivariado de clasificación (clúster), con el cual se determinó la correlación existente entre los parámetros analizados y la estructura del suelo, a fin de relacionarla con los posibles comportamientos ambientales, de modo que pueden ser tratados como insumos en diagnósticos ambientales de suelos.

\section{Resultados}

Se tomaron 120 muestras de suelos de manera aleatoria, las cuales se categorizaron bajo el sistema de clasificación de suelos USCS, encontrándose que el mayor porcentaje de su composición corresponde a arcillas de alta plasticidad $(\mathrm{CH})$, tal como lo indica la Figura 1. Además, de acuerdo con su naturaleza, el mayor porcentaje corresponde a suelos cohesivos, con un $77 \%$, y granulares $(23 \%)$. 

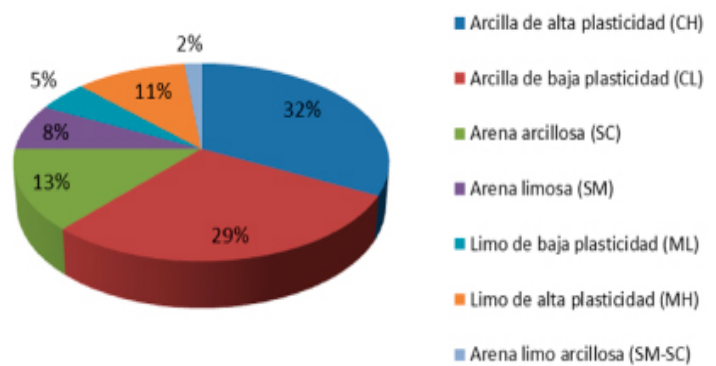

Figura 1.

Distribución porcentual

de muestras

estudiadas.

Fuente: Autor, 2016.

A las muestras se les realizaron también los análisis de humedad natural, peso unitario (densidad real), materia orgánica y carbono orgánico, y se agruparon de acuerdo con la naturaleza de su conformación y estructura USCS. Mediante un análisis estadístico descriptivo multivariado de clasificación (clúster), se determinó la correlación por el método jerárquico de aglomeración que se muestra en la Figura 2. En este contexto, donde las características físico-químicas son las variables de agrupación, este tipo de representación resulta siendo una descripción sintética del comportamiento sinérgico en el suelo de las características físico-químicas analizadas, y evidencia las relaciones de cercanía y dependencia entre las variables de agrupación. Dicha cercanía estadística valida las propiedades e interacciones que se encuentran en la literatura sobre las mismas.

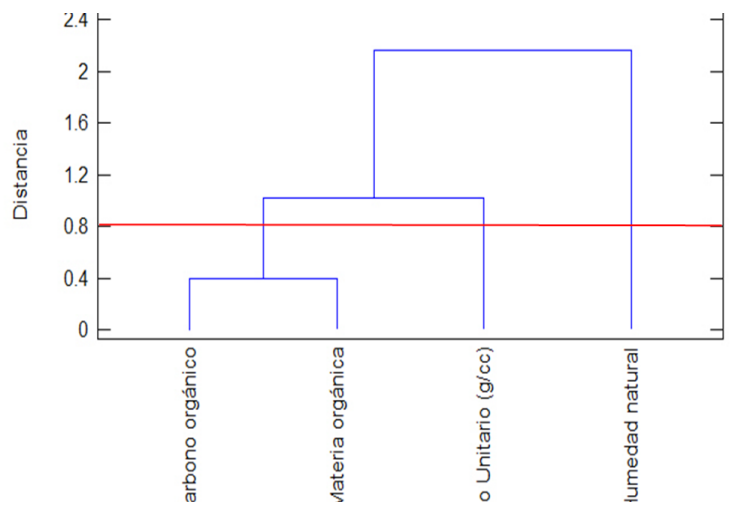

\author{
Figura 2. \\ Dendrograma de análisis \\ de clasificación (cluster) \\ por método jerárquico de \\ aglomeración, generado \\ mediante método de ward, con \\ métrica de distancia euclideana \\ y conglomerados a partir de las \\ características físico químicas \\ como variables. \\ Fuente: Autor, 2016.
}

Más concretamente, el dendrograma de la Figura 2 muestra la formación de tres clúster: uno conformado por los porcentajes de Materia orgánica del suelo (MOS) y Carbono orgánico del suelo (COS); otro que une el anterior con la característica de peso unitario: y un tercero que relaciona todos los anteriores con la característica de porcentaje de humedad natural.

Las relaciones entre la materia orgánica, expresada como variables mediante el \%MOS y \%COS, el peso unitario o densidad aparente y la humedad natural en el presente estudio, son explicadas por Zambrano, Apráez y Navia [6], quienes encontraron en su estudio que los valores de densidad aparente están directamente relacionados con los altos contenidos de $\mathrm{MO}$, debido a que está última hace disminuir la densidad al incrementar la estabilidad de los agregados del suelo y ocupa un menor volumen en comparación con el mineral propio del suelo [7]; a su vez, lo anterior permite una mayor retención de agua [6]. 
Al ser el primer eslabón de agrupación, el primer clúster formado, que relaciona los porcentajes de MOS y COS, representa una marcada susceptibilidad a variables externas no evaluadas dentro del análisis clúster, que en este caso refiere a los factores ambientales propios del entorno natural y/o antrópico de los suelos muestreados. Por ejemplo, si se considerara un suelo prístino, los niveles de MOS serían regulados por el clima, pues este afecta las entradas y salidas del balance de carbono en el suelo. Esto ha sido reportado por autores como Sainz Rozas, Echeverria y Angelini [1], al asociar la caída de los niveles de MOS con la disminución de la precipitación media anual y el aumento de la temperatura media; y su incremento, con el aumento de la proporción de arcilla en el suelo y con la mayor disponibilidad de agua en el entorno.

Ello también representa una relación de homogeneidad, dado que el COS es el principal elemento de la MOS, y cuando esta asociación se da, le proporciona al suelo coloides [8] que influye directamente en sus propiedades físicas, químicas y biológicas. Igualmente, hacen parte de sus propiedades bioorgánicas, y es tal su importancia en el comportamiento del mismo que son ampliamente tenidos en cuenta en su clasificación taxonómica.

En cualquier caso, el aumento de la MOS en la estructura del suelo favorece su formación e incrementa el tamaño y estabilidad de los agregados; también aumenta la cantidad de macroporos, el volumen de aireación y la capacidad de retención de agua [9].

Asimismo, el COS es reconocido como uno de los principales indicadores de la calidad de suelo [5], ya que favorece la agregación y distribución del espacio poroso, y condiciona directamente la humedad aprovechable, la retención y el movimiento del agua, y la difusión de gases en el suelo, todo lo cual aumenta la capacidad de intercambio catiónico (CIC), la capacidad buffer y la retención de fosfatos [10].

Se espera entonces que la textura tenga un impacto directo en las entradas y salidas de MOS, e indirecto sobre la productividad de la vegetación a través de la disponibilidad de agua y la fertilidad del suelo. Todo esto ocurre gracias a la asociación coloidal de la MOS con las arcillas, que define la tasa de descomposición del Carbono orgánico del suelo (COS) [11,12].

Lo anterior se evidencia, por demás, en los comportamientos individuales de las muestras recuperadas según la naturaleza de su conformación y estructura USCS en los ensayos realizados (Figura 3). Por ello en estas muestras se encuentran valores de material orgánico promedio que varían entre 6 y 34\%, así como carbono orgánico con promedio, entre el 10 y 59\%. De todos modos, los suelos cohesivos presentan mayores contenidos de ambas características, dada su mayor afinidad al material orgánico (MOS y COS). Sin embargo este factor es susceptible a variación, pues depende, como ya se ha mencionado, de otros factores ambientales como la presencia de vegetación, características biológicas, variación de temperatura, entre otros. 


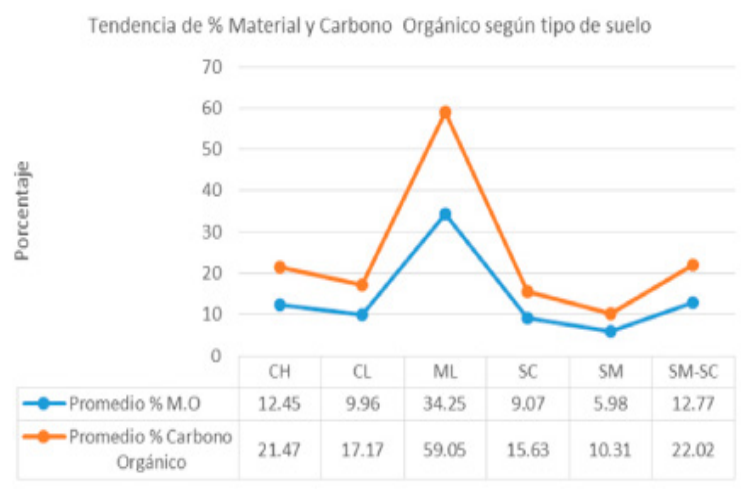

Figura 3.

Tendencia en porcentaje de material orgánico.

Fuente: Autor, 2016.

El segundo y tercer clúster por su parte representan una relación de dependencia con sus respectivos predecesores que se explicará a continuación; y una relación de heterogeneidad entre sí, dado que el primero significó una agrupación de propiedades bio-orgánicas, mientras que la característica de peso unitario hace parte de las propiedades físicas del suelo en su fase sólida y la característica de humedad natural, de las propiedades físicas del suelo pero en su fase líquida, contrastando así total y parcialmente con el primero y segundo clúster, respectivamente. Esto último se evidencia cuantitativa y proporcionalmente en el rango de separación de la escala vertical representada en la Figura 2.

Sobre la característica de peso unitario o densidad aparente se sabe que la medición permite estimar las condiciones de estructura del suelo, bien sea utilizándolo como un parámetro para estimar el grado de compactación, toda vez que si esta condición se presenta la densidad aparente se incrementa, o bien como un indicador del contenido de materia orgánica en el suelo, puesto que esta reduce el valor de dicha densidad [9]; además esta situación da indicios sobre el tamaño de las fracciones granulométricas presentes, pues los valores de densidad aparente guardan una relación inversa con el tamaño de partícula del suelo [13]. Al respecto, [14] ya se había mencionado que la densidad aparente podría ser evaluada como un indicador de la estructura y dureza del suelo para monitorear su calidad.

En general, tales condiciones se observan en los comportamientos individuales de las muestras estudiadas de acuerdo con su conformación y estructura USCS para la característica de peso unitario (Figura 4), para la cual se encontraron valores promedio entre 1 y $1.9 \mathrm{~g} / \mathrm{cc}$.

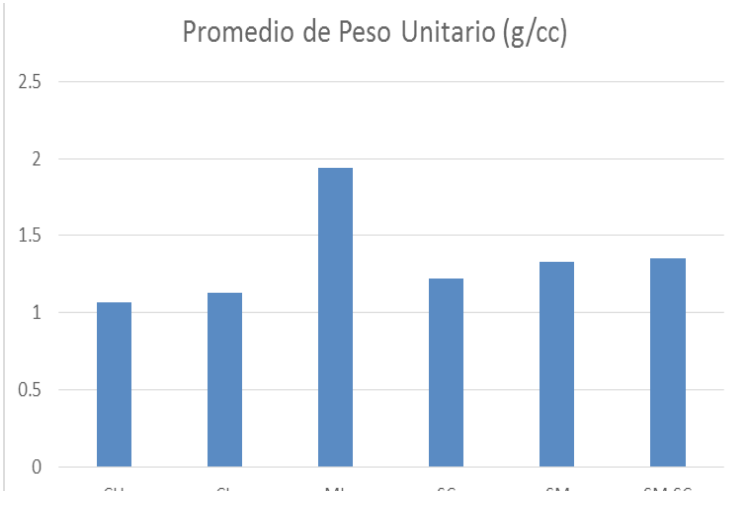

Figura 4.

Peso unitario (densidad) promedio de acuerdo a clasificación USCS. Fuente: Autor, 2016. 
Sobre esta fluctuación de valores, Engaramo, Paz Ferreiro, Mirás Avalos y Vidal Vázquez [15] manifiestan que las mediciones de densidad aparente en el suelo son altamente variables y dependen de factores como la textura, estructura, contenido de materia orgánica, arcilla y humedad, o del tipo de manejo dado al suelo, lo que a su vez condiciona sus características de retracción, expansión, dureza o resistencia mecánica y porosidad. Esto últimoes corroborado por Volverás Mambuscay, Amézquita Collazos y Campo Quesada [16], los cuales, además de encontrar que los valores de densidad aparente están directamente relacionados con los niveles de materia orgánica, evidenciaron que la densidad aparente tiende a aumentar con el tiempo e intensidad de uso del suelo, ya que disminuye la estabilidad estructural y genera pérdidas de partículas finas y volumen de suelo. es así como las partículas removidas y sueltas se reacomoden y ocupan los espacios o poros ahora libres [17].

Ahora bien, la característica de humedad natural se ve mayormente influenciada por el diámetro de las partículas del suelo que por la fuente o yacimiento del sustrato, de manera que existe una relación inversa entre el tamaño de las fracciones granulométricas y el contenido relativo de agua en el suelo [13]. Asimismo, dicha humedad se relaciona con otras variables del suelo, como la textura y la densidad aparente [18], en tanto sus propiedades físicas determinan el proceso de retención agua; esto debido a que la textura relaciona el tamaño de las partículas del suelo y la superficie específica de reacción, en tanto que la densidad aparente se ve marcada por la cantidad de poros por centímetro cúbico presentes en el suelo [7].

Para ambas propiedades, el efecto del agua en el suelo se da en su almacenamiento y movimiento y depende de las proporciones de arcilla o arena $[19,20]$. Aunque generalmente se mira a partir del contenido de arcilla dado que estas partículas tienen mayor capacidad de retención y almacenamiento de agua en comparación con los limos y las arenas [21, 22].

Lo anterior se confirmó con el comportamiento individual de las muestras para la característica de humedad natural, que se muestra en la Figura 5. Al respecto, es preciso anotar que las muestras de arena limo arcillosas no quedaron incluidas en la figura, pues corresponden a un caso frontera y no hay suficientes muestras para que constituyan un aporte significativo a la investigación y que la clasificación de limos de alta y baja plasticidad representan suelos limosos con condiciones variables y adicionales de partículas de arcilla en equilibrio estructural. Por esta razón se espera de ellas un comportamiento superior al de las muestras de suelo netamente arcillosas.

Como se observa, los valores obtenidos van entre 15\% y 39\%, y los mayores porcentajes corresponden a suelos de naturaleza cohesiva. Esto puede atribuirse a que el carácter cohesivo permite retener mayor contenido de agua en su estructura, mientras que los suelos granulares propician mayor infiltración y menor retención de agua. 


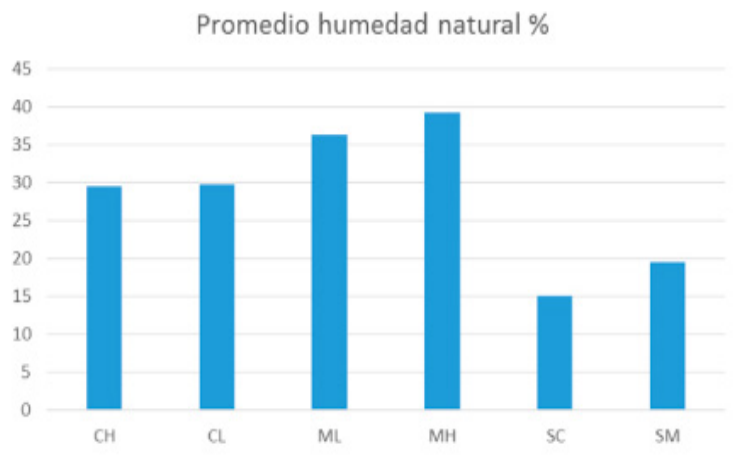

Figura 5.

Humedad natural promedio de acuerdo con la clasificación USCS.

Fuente: Autor, 2016.

Teniendo claridad sobre la influencia de las características analizadas en los procesos evolutivos del suelo, un análisis clúster, esta vez usando como elementos de agrupación la clasificación USCS de las muestras a partir de sus características físico-químicas, evidenció la relación entre la génesis del suelo, es decir, sus aspectos naturales de conformación y dichas características, como se muestra en la Figura 6. Por su parte, esta representación da fe de las similitudes existentes entre las muestras de suelo con base en sus características físico-químicas.

La agrupación observada muestra que las características naturales de conformación y estructura USCS del suelo se derivan de sus características físico-químicas, de manera que las muestras pertenecientes a un mismo grupo son semejantes entre sí. A esto se añade que las muestras pertenecientes a grupos diferentes tienen un comportamiento distinto con respecto a las variables analizadas, lo que se evidencia cuantitativa y proporcionalmente en el rango de separación de la escala vertical de la misma Figura 6.

A tono con todo lo antes planteado, en el dendrograma de la Figura 6 se percibe una partición en grupos o clúster en la que el primero reúne las muestras de naturaleza cohesiva ( $\mathrm{CH}$ y CL); el segundo, las granulares (SC, SM y SM-SC); y el tercero, la condición de frontera entre los anteriores (ML). Al cruzar este resultado de agrupación y los de la clasificación (clúster) de las características físico-químicas se infiere que el criterio edáfico que logró la agrupación más consistente es el contenido de materia orgánica.

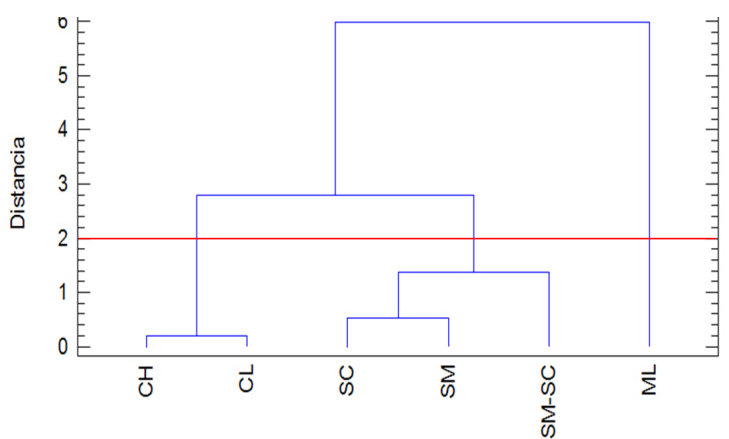

\section{Figura 6.}

Dendograma de análisis de clasificación (cluster) por método jerárquico de aglomeración, generado mediante método de ward, con métrica de distancia euclideana y conglomerados a partir de la clasificación USCS de las muestras de suelo.

Fuente: Autor, 2016. 
Esta tendencia de agrupación en función de las características físico-químicas coincide con la trayectoria de desarrollo edáfico encontrada en un estudio de correlación múltiple de diez propiedades edáficas cuantitativas, en el que se halló en concreto que la materia orgánica es un atributo físico-químico que se correlaciona de manera positiva con los porcentajes de porosidad, arcilla y humedad y en forma negativa con la densidad aparente y real; con los porcentajes de arena y limo, así como con la compactación y los valores de $\mathrm{pH}$ [23]. En el estudio cuestión, las propiedades edáficas responsables de agruparse como sitios similares fueron la materia orgánica, la densidad real, los porcentajes de porosidad y de arena, así como la densidad aparente.

Este análisis de agrupación (clúster) a partir de la clasificación USCS de las muestras de suelo también muestra que las características físico-químicas analizadas influyen en la distribución, cantidad y tipo de partículas que conformarán la textura del suelo, dado que en la Figura 6 se evidencia una agrupación más consistente cuando la presencia de texturas arcillosas es predominante. Lo contrario ocurre cuando dominan texturas arenosas, lo cual es un indicador de disturbio debido a que se alteran en mayor medida los patrones de distribución y agrupación de las muestras de suelo.

En este análisis no se mencionan las características del tercer clúster porque, como ya se había mencionado, corresponde a una condición de frontera entre los otros dos, conformada sin una dominancia marcada de texturas arcillosas o arenosas. No obstante, dada la separación vertical en el dendograma, se esperaría que el comportamiento ambiental del tercer clúster tuviera más afinidad con el segundo que con el primero; en este sentido, se requieren estudios de campo más amplios y detallados, que puedan marcar una tendencia con mayor precisión y exactitud. A pesar de todo, la cercanía puede ser atribuida a las características estructurales y en términos de retención hídrica, ya que en la ciencia del suelo el término arcilla tiene 2 significados: 1) toda partícula del que tenga un tamaño menor de $2 \mathrm{~mm}$ independientemente de su composición química, a veces incluyendo la fracción limo entre 50 y 2 m; y 2) al mineral formado por la unión de 1 o 2 láminas tetraédricas con un lámina octaédrica. Hoy en día también se consideran como arcillas los óxidos y oxi hidróxidos, abundantes en suelos fuertemente meteorizados y los materiales "amorfos" conocidos como minerales de corto rango de cristalinidad y que pueden tener varias formas: las alofanas son esferas y las imogolitas son tubos, pero no dan señal en los difracto gramas de rayos X [24]. En este sentido, numerosos trabajos han demostrado que la textura es una característica de los suelos que influyen más en su capacidad de retención hídrica. Asimismo la combinación de variables texturales, contenidos en carbono orgánico y densidad aparente han proporcionado ecuaciones que predicen bastante bien algunos puntos característicos de la curva de retención hídrica de los suelos [25]

De acuerdo con lo anterior, comparando el primer clúster, formado entre las muestras $\mathrm{CH}$ y $\mathrm{CL}$, con el segundo, formado entre las muestras de SC, SM y SM-SC, se esperaría como comportamiento ambiental consecuente que los primeros sean un suelo de mayor dureza y resistencia, poca infiltración, mayor retención de la humedad, de agregados y estructura más estable, así como mayor contenido de materia orgánica, con propensión a la retracción y expansión, y una mayor proporción de material fino. 


\section{Conclusión}

Basados en los resultados de la clasificación y agrupación de las muestras de suelo analizadas conforme al sistema taxonómico USCS en función de las características físico-químicas estudiadas, las agrupaciones reportadas corroboran la existencia de características físico-químicas del suelo que permiten correlacionar comportamientos ambientales con condiciones edafogénicas.

Desde una valoración cuantitativa y estadística, esto sugiere la existencia de procesos edafogénicos con trayectorias diferenciales atribuibles a características físico-químicas del suelo que dan lugar a comportamientos ambientales particulares. En este sentido, es importante un análisis integral de las características físico-químicas del suelo como criterios edáficos para su clasificación para orientar su ordenamiento ambiental respecto a su uso, manejo y ocupación, todo lo cual deberá ser coherente con los comportamientos ambientales que dichas características denotan.

Además, de acuerdo con los ensayos realizados y el comportamiento correlacionado con las características físico-químicas, los posibles cambios y comportamientos de las características descritas, se infiere la necesidad de implementar prácticas de manejo que preserven los procesos edafogénicos para revertir y evitar procesos de degradación. En este contexto, la densidad aparente o peso unitario, junto a la granulometría y retención de humedad resultan un parámetro útil para estimar el deterioro del suelo, en tanto el contenido de materia orgánica es un criterio edáfico para la clasificación y agrupación de las muestras de suelo, dada su influencia directa e indirecta sobre las demás características físico-químicas del suelo. 


\section{Referencias}

1 H. R. Sainz Rozas, H. E. Echeverria, and H. P. Angelini, “Niveles de carbono orgánico y ph en suelos agrícolas de las regiones pampeana y extrapampeana argentina," Ciencias del Suelo, vol. 29, no. 1, pp. 29-37, 2011.

2 F. Hupet and M. Vanclooster, "Intraseasonal dynamics of soil moisture variability within a small agricultural maize cropped field," Journal of Hydrology, vol. 261, no. 1-4, pp. 86-101, Apr. 2002.

3 M. E. Hawley, T. J. Jackson, and R. H. McCuen, "Surface soil moisture variation on small agricultural watersheds," Journal of Hydrology, vol. 62, no. 1-4, pp. 179-200, Apr. 1983.

$4 \quad$ R. B. Grayson and A. W. Western, "Towards areal estimation of soil water content from point measurements: time and space stability of mean response," Journal of Hydrology, vol. 207, no. 1-2, pp. 68-82, Jun. 1998.

$5 \quad$ M. Eyherabide, H. Saínz Rozas, P. Barbieri, and H. E. Echeverría, “Comparación de métodos para determinar carbono orgánico en suelo," Ciencias del Suelo, vol. 32, no. 1, pp. 13-19, 2014.

6 G. Zambrano B., J. E. Apráez G., y J. F. Navia E., “Relación de las propiedades del suelo con variables bromatológicas de Pastos, en un sistema lechero de Nariño," Revista de Ciencias Agrícolas, vol. 31, no. 2, pp. 106-121, Dec. 2014.

$7 \quad$ L. Thompson and F. Troeh, Los suelos y su fertilidad, 4a ed. Reverté, 1980.

8 E. Martínez H, J. P. Fuentes E, and E. Acevedo H, "Carbono orgánico y propiedades del suelo," Revista de la ciencia del suelo y nutrición vegetal, vol. 8, no. 1, pp. 68-96, 2008.

9 D. F. Jaramillo J., Introducción a la ciencia del suelo. Medellín: Universidad Nacional de Colombia, 2002.

10 M. L. Gutiérrez, "Carbono como indicador de degradación de la calidad del suelo bajo diferentes coberturas en el páramo de Guerrero," Universidad Nacional de Colombia, Bogotá, Colombia, 2015.

11 Z. X. Tan, R. Lal, N. E. Smeck, and F. G. Calhoun, “Relationships between surface soil organic carbon pool and site variables," Geoderma, vol. 121, no. 3-4, pp. 187-195, 2004.

12 S. Wang, X. Wang, and Z. Ouyang, "Effects of land use, climate, topography and soil properties on regional soil organic carbon and total nitrogen in the Upstream Watershed of Miyun Reservoir, North China," Journal of Environmental Sciences, vol. 24, no. 3, pp. 387-395, 2012.

13 P. Vargas Tapia, J. Z. Castellanos Ramos, J. de J. Muñoz Ramos, P. Sánchez García, L. Tijerina Chávez, R. M. López Romero, C. Martínez Sánchez, and J. L. Ojodeagua Arredondo, “Efecto del tamaño de partícula sobre algunas propiedades físicas del tezontle de Guanajauto, México," Agricultura técnica en México, vol. 34, no. 3, pp. 323-331, 2008.

14 J. W. Doran and T. B. Parkin, "Defining and assessing soil quality," in Defining soil quality for a sustainable environment, 1994, pp. 3-21.

15 O. E. Ingaramo, J. Paz Ferreiro, J. M. Mirás Avalos, and E. Vidal Vázquez, "Caracterización de las propiedades generales del suelo en una parcela experimental con distintos sistemas de laboreo," Cadernos do Laboratorio Xeolóxico de Laxe, vol. 32, pp. 127-137, 2007. 
16 B. Volverás Mambuscay, É. Amézquita Collazos, and J. M. Campo Quesada, “Indicadores de calidad física del suelo de la zona cerealera andina del departamento de Nariño, Colombia," Corpoica Ciencia y Tecnología Agropecuaria, vol. 17, no. 3, pp. 361-377, Aug. 2016.

17 N. C. Gutiérrez, C. A. Venialgo, J. R. Gutiérrez, D. M. Drganc, and J. D. Oleszczuk, “Efecto del manejo del suelo sobre la densidad aparente y la resistencia mecánica a la penetración," Universidad Nacional del Nordeste - UNNE - Comunicaciones Científicas y Tecnológicas, 2000.

18 K. Largaespada and C. Henríquez, "Distribución espacial de la humedad y su relación con la textura en un suelo," Agronomía Costarricense, vol. 39, no. 3, pp. 137-147, 2015.

19 J. Porta, M. López, and C. Roquero, Edafología para la agricultura y el medio ambiente. España: S.A. MUNDI-PRENSA LIBROS, 2003.

20 C. Henríquez and G. Cabalceta, Guía práctica para el estudio introductorio de los suelos con un enfoque Agrícola, 2a Ed. San José, Costa Rica: Universidad de Costa Rica, 2012.

21 S. Gavande, Física de suelos: Principios y aplicación, 1a Reimp. México D. F., México: LIMUSA-WILEY S.A., 1973.

22 R. Honorato, Manual de Edafología, 4a Ed. México: ALFAOMEGA, 2000.

23 I. Castellanos Vargas, N. E. García Calderón, and Z. Cano Santana, "Procesos físicos del suelo en la reserva ecológica del Pedregal de San Ángel de Ciudad Universitaria: atributos para su conservación," Terra Latinoamericana, vol. 35, no. 1, pp. 51-64, 2017.

24 A. Alvarado, R. Mata, and M. Chinchilla, "Arcillas identificadas en suelos de Costa Rica a nivel generalizado durante el período 1931-2014: II. mineralogía de arcillas en suelos con características vérticas y oxídico caoliníticas," Agronomía Costarricense, vol. 38, no. 1, pp. 107-131, 2014.

25 C. Boix, A. Calvo Cases, J. M. Schoorl, and M. D. Soriano Soto, "Influencia de la estructura y usos del suelo en las características de retención hídrica de suelos mediterráneos sobre litología caliza," Cadernos do Laboratorio Xeoloxico de Laxe, vol. 21, pp. 587-596, 1996.

Como citar este artículo: C. Díaz, C. Herrera, y K. Prada, "Características físico químicas de suelos con relación a su conformación estructural",Investigación e Innovación en Ingenierias, vol. 6, n. 1, pp. 58 - 69., 2018. DOl: https://10.17081/invinno.6.1.2775 